\title{
COMPARAÇÃO ENTRE DOSES DE ALIL ISOTIOCIANATO E A SOLUÇÃO DE FORMALDEÍDO NA EXTRAÇÃO DE MINHOCAS ( $\left.{ }^{1}\right)$
}

\author{
ROBINSON ROLIM RESSETTI $(2 *)$; JAIR ALVES DIONÍSIO ( $\left.{ }^{3}\right)$; ANTONIO CARLOS VARGAS MOTTA ( ${ }^{3}$ )
}

\begin{abstract}
RESUMO
A solução de formaldeído é o extrator mais utilizado na amostragem de minhocas, mesmo com riscos potenciais aos seres humanos e ao meio ambiente. O reagente Alil isotiocianato (AITC), fitocomplexo da mostarda sintetizado em laboratório, foi testado em condições de clima temperado. Entretanto, não existem dados de sua utilização no Brasil. Esta pesquisa teve por objetivo avaliar a eficácia do reagente AITC em relação à solução de formaldeído 2,2 $\mathrm{g} \mathrm{L}^{-1}$ na extração de minhocas, sob diferentes sistemas de uso do solo no início do verão. $\mathrm{O}$ experimento foi realizado em uma fazenda localizada no Município de Castro (PR), no período de 16 a 23 de dezembro de 2003. Os três sistemas selecionados foram: 1) um talhão de plantio direto também utilizado para pastejo; 2) pastagem perene; e 3) mata subtropical perenifólia. No talhão de plantio direto, o AITC $50 \mathrm{mg} \mathrm{L}^{-1}$ resultou em maior eficiência na obtenção da densidade populacional (144 indivíduos $\left.\mathrm{m}^{-2}\right)$ e biomassa $\left(26,3 \mathrm{~g} \mathrm{~m}^{-2}\right)$ do que a solução de formaldeído $\left(123,4\right.$ ind $\mathrm{m}^{-2}$ e $21,7 \mathrm{~g} \mathrm{~m}^{-2}$ respectivamente). Na pastagem perene e na mata, a solução de formaldeído foi de maior eficácia. Foram encontradas minhocas das famílias Megascolecidae, Octochaetidae e Lumbricidae. O AITC é considerado promissor na determinação da densidade populacional e biomassa em sistemas de plantio direto. Os resultados variam com a concentração da solução extratora e o sistema avaliado.
\end{abstract}

Palavras-chave: minhoca, extração, formaldeído, AITC, densidade, biomassa.

\section{ABSTRACT \\ DETERMINATION OF ALLYL ISOTHIOCYANATE CONCENTRATION TO SUBSTITUTE FORMALDEHYDE FOR SAMPLING EARTHWORMS}

Formaldehyde solution is the commonest substance used in the world to extract earthworm from soil despite of its harmful potential to humans and for the environment. Allyl isothiocyanate (AITC), a mustard oil, was tested under temperate climate condition. There is no record of its use on Brazilian soils. This research was established to evaluate the performance of different AITC rates compared to formaldehyde, under different soil uses at early summer. The experiment was established in a farm located at Castro county, Paraná State, Brazil, from 16 to 23 of December, 2003. Three ecosystems were selected: 1) no-tillage field used for crop-livestock; 2) permanent grassing pasture; and 3) araucaria native forest. In no-tillage, AITC $50 \mathrm{mg} \mathrm{L}^{-1}$ presented the highest density (144 individuals $\mathrm{m}^{-2}$ ), followed by formaldehyde $\left(123.4 \mathrm{~m}^{-2}\right)$, AITC $100\left(115.4 \mathrm{~m}^{-2}\right)$ and AITC $150\left(76.1 \mathrm{~m}^{-2}\right)$. For biomass, AITC 50, 100 and $150 \mathrm{mg} \mathrm{L}^{-1}$ extraction were superior to formaldehyde $\left(21.7 \mathrm{~g} \mathrm{~m}^{-2}\right)$. In permanent pasture, formadehyde gave three and two fold more density and biomass, respectively than the other treatments. In the forest, formaldehyde was superior to the AITC rates tested. However, AITC 50 and $100 \mathrm{mg} \mathrm{L}^{-1}$ were equivalent to formaldehyde for biomass. Megascolecidae, Octochaetidae and Lumbricidae were the three earthworm' families found in the experimental area. The use of AITC is prospective to determine density and biomass under no-till system. The results vary with extractor concentration and ecosystem.

Key words: earthworm, extraction, formaldehyde, AITC, density, biomass.

$\left({ }^{1}\right)$ Parte da Dissertação de Mestrado do primeiro autor apresentada ao Curso de Pós-Graduação em Ciência do Solo da Universidade Federal do Paraná (UFPR). Recebido para publicação em 16 de dezembro de 2005 e aceito em 29 de agosto de 2007.

$\left({ }^{2}\right)$ Engenheiro Agrônomo, Especialista em Gerenciamento Ambiental na Indústria (DEQ-UFPR). Avenida República do Líbano, 342, Jardim Social, 82520-500 Curitiba (PR). E-mail: robinsonrr@onda.com.br $\left(^{*}\right)$ Autor correspondente.

$\left({ }^{3}\right)$ Departamento de Solos e Engenharia Agrícola da UFPR, Rua dos Funcionários, 1534, 80035-050 Curitiba (PR). E-mails: jair@ufpr.br; mottaacv@ufpr.br. 


\section{INTRODUÇÃO}

As minhocas participam na formação e no desenvolvimento da maior parte dos solos desde que surgiram nesse planeta há 600 milhões de anos, atuando na constituição e conservação de sua fertilidade natural (SINNAEVE, 1999).

Existem diferentes métodos físicos e químicos para monitorar a qualidade do solo, mas o sistema vivo (qualidade biológica) deve ser avaliado por parâmetros biológicos (RöMBKE e KALSCH citados por Ruf et al., 2003). A densidade populacional e biomassa de minhocas são influenciadas pelos fatores climáticos e propriedades intrínsecas do solo, cobertura vegetal, práticas agrícolas, intensidade de mecanização, manejo dos resíduos, utilização de fertilizantes químicos, defensivos agrícolas, adubação verde e orgânica entre outras (EDWARDS, 1983). Por essas razões as minhocas são consideradas excelente indicador do tipo de uso e da qualidade do solo (Paoletti, 1999).

Existem diferentes métodos de amostragem para avaliar a densidade populacional e biomassa de minhocas terrestres. Os dois métodos mais utilizados são: a coleta manual e a extração por soluções irritantes. Ambos apresentam vantagens e desvantagens (LeE, 1985a; Satchell citado por GunN, 1992 e ZABORSKI, 2003). A coleta manual limita a captura das minhocas que se encontram na camada amostrada. Esse método também não pode ser aplicado em áreas com barreiras físicas, como pedras e minerais de grandes dimensões e também sistema radicular denso ou lenhoso.

A extração por meio químico, que envolve a irrigação do solo com uma solução irritante, com posterior coleta das minhocas que emergem à superfície (RAW, 1959; Evans e Guild citados por LeE, 1985a e ZABORSKI, 2003) é um método mais rápido e simples, podendo ser utilizado em diferentes condições. Um dos extratores químicos de minhocas mais utilizados é uma solução de formaldeído (ZABORSKI, 2003), mas a eficiência do método é influenciada pela concentração da solução, temperatura e umidade no solo (RAw, 1959; Lakhani e Satchell citados por ZABORSKI, 2003). Outra desvantagem do método de extração com formaldeído é que esse produto é fitotóxico e carcinogênico e a legislação pode vir a impedir sua utilização (SCHMIDT et al., 2001).

Como alternativa à utilização de formaldeído, é possível a utilização de uma suspensão de pó de mostarda comercial, minimizando os danos ao ambiente e à saúde do aplicador (GunN, 1992). Todavia, apenas com a utilização de um reagente de composição conhecida e constante é que se pode padronizar a dose a ser utilizada.

Zaborski (2003) testou o reagente Alil isotiocianato (AITC) PA na extração de minhocas. O AITC é um dos fitocomplexos presentes em tecidos de crucíferas (Borek et al., 1995) e exerce um efeito irritante nas minhocas terrestres

Neste trabalho, compararam-se doses diferentes de AITC para maximizar a expulsão de minhocas do solo, visando substituir a solução de formaldeído 2,2 $\mathrm{g} \mathrm{L}^{-1}$ (RAW, 1959) na extração de minhocas terrestres nas condições da região do estudo.

\section{MATERIAL E MÉTODOS}

A extração de minhocas foi realizada no período de 16 a 23 de dezembro de 2003. O experimento foi realizado na Fazenda Onça, filiada à Cooperativa Castrolanda na Colônia Castrolanda, Município de Castro (PR). A propriedade selecionada explora a agropecuária, predominantemente de suínos. Os resíduos gerados nessa atividade são utilizados na produção de grãos e na pastagem.

A sede da fazenda possui as coordenadas geográficas de $24^{\circ} 48^{\prime} 00^{\prime \prime}$ latitude Sul e $49^{\circ} 54^{\prime} 05^{\prime \prime}$ longitude Oeste e altitude de $1.000 \mathrm{~m}$. O município de Castro situa-se, na maior parte, no primeiro planalto paranaense, com uma pequena parte a oeste no segundo planalto. O clima da região é $\mathrm{Cfb}$ segundo Köppen, temperado úmido, mesotérmico, com temperatura média anual em torno de $17{ }^{\circ} \mathrm{C}$ e precipitação pluvial anual média entre 1.400 e 1.600 mm (IAPAR, 2000; FAsOLO et al., 2002).

Os sistemas avaliados foram: plantio direto, pastagem perene e mata. O talhão de plantio direto, de 36,54 ha, seguia a sucessão soja-milho-feijão, com azevém (Lolium multiflorum) como cobertura para o feijão (Phaseolus vulgaris) e soja (Glycine max), e aveia (Avena sp.) ou cevada (Hordeum vulgare) como cobertura para o milho (Zea mays). Na época de extração das minhocas, havia sido colhido o trigo (Triticum sp.) utilizado para pastejo, produção de grãos e retirada da palha para feno e a cultura da soja estava recém-implantada. Entretanto, a cultura do trigo não é explorada com muita freqüência.

A pastagem perene, de 25 ha, era composta de Brachiaria sp., Cynodon sp. e gramíneas nativas, sendo utilizada por bovinos e ovinos. A área de mata era de preservação permanente e com relevo ondulado, constituindo proteção para um corpo de água (mata ciliar) e tendo como vegetação primária floresta subtropical perenifólia. 
Os solos da região são do Grupo Campos Gerais da Formação Ponta Grossa, tendo como material de origem, fundamentalmente, folhelho. $\mathrm{O}$ relevo regional é suave ondulado. Os solos predominantes são Latossolo Vermelho distrófico húmico, Cambissolo Háplico tb distrófico típico e Latossolo Bruno ácrico húmico (FAsolo et al., 2002).

Para a caracterização do solo nos sistemas estudado foram coletadas vinte amostras simples próximas aos locais de extração das minhocas, na camada de 0-15 cm para formar uma composta por ecossistema avaliado. As análises químicas e granulométricas foram realizadas nos Laboratórios de Fertilidade e Física do Solo da UFPR segundo Marques e Motta (2003) e Machado (2003), respectivamente. Os resultados das análises químicas e físicas encontramse nas tabelas 1 e 2 .

\subsection{Extração com solução de formaldeído a $2,2 \mathrm{~g} \mathrm{~L}^{-1}$ e AITC nas doses de 50, 100 e $150 \mathrm{mg} \mathrm{L}^{-1}$}

A amostragem dos diferentes ecossistemas foi realizada em locais com distância mínima entre si de 5 m (Bouché e Gardner, 1984) seguindo quatro linhas paralelas no talhão de plantio direto (uma por extrator), duas linhas paralelas na pastagem perene (dois extratores por linha) e totalmente aleatória na mata.

Tabela 1. Caracterização química dos solos nos sistemas plantio direto, pastagem perene e mata, na profundidade de 0-15 cm, Castro (PR), 2003

\begin{tabular}{lccccccccccc}
\hline Sistemas & $\mathrm{pH} \mathrm{CaCl} 2$ & $\mathrm{Al}^{3+}$ & $\mathrm{H}+\mathrm{Al}$ & $\mathrm{Ca}^{2+}$ & $\mathrm{Mg}^{2+}$ & $\mathrm{K}^{+}$ & $\mathrm{T}$ & $\mathrm{P}$ & $\mathrm{C}$ & $\mathrm{m}$ & $\mathrm{V}$ \\
\hline & & & & & $\mathrm{cmol}_{\mathrm{c}}$ & $\mathrm{dm}^{-3}$ & & & $\mathrm{mg} \mathrm{dm}^{-3}$ & $\mathrm{~g} \mathrm{dm}^{-3}$ & $\%$ \\
Plantio direto & 6,0 & 0,0 & 2,5 & 6,1 & 3,6 & 0,74 & 13 & 11,5 & 31,7 & 0,0 & 80,7 \\
Pastagem & 5,8 & 0,0 & 3,7 & 6,8 & 4,9 & 0,87 & 16,2 & 636 & 39,5 & 0,0 & 77,2 \\
Mata & 4,3 & 1,4 & 10,2 & 5,0 & 3,2 & 0,35 & 18,7 & 45 & 60,6 & 14,1 & 45,6 \\
\hline
\end{tabular}

Tabela 2. Análise granulométrica dos solos nos ecossistemas plantio direto, pastagem perene e mata, na profundidade de 0-15 cm, Castro (PR), 2003

\begin{tabular}{lccc}
\hline Sistemas & Areia & Silte & Argila \\
\cline { 2 - 4 } & & $\mathrm{g} \mathrm{kg}^{-1}$ & \\
Plantio direto & 200 & 320 & 480 \\
Pastagem & 240 & 280 & 480 \\
Mata & 220 & 300 & 480 \\
\hline
\end{tabular}

Os resíduos da superfície do solo foram retirados manualmente nas áreas de plantio direto e mata e com o auxílio de uma enxada na área de pastagem, fixando-se em seguida um anel metálico com área de $0,1301 \mathrm{~m}^{2}$ e altura de $0,108 \mathrm{~m}$ onde foram adicionadas as soluções extratoras. $O$ anel foi aprofundado manualmente no solo para evitar o escoamento da solução extratora para seu exterior (CHAN e MunRo, 2001). As soluções extratoras utilizadas foram formaldeído a 2,2 $\mathrm{g} \mathrm{L}^{-1}$ (RAW, 1959) e AITC nas doses de 50, 100 e $150 \mathrm{mg} \mathrm{L}^{-1}$ (Zaborski, 2003). Foi utilizado o reagente Allyl isothiocianate marca Fluka (pureza $\geq 98 \%$ e densidade 1,02 $\mathrm{g} \mathrm{mL}^{-1}$ ), que não é prontamente solúvel em água. Assim, foi diluído em isopropanol a fim de se obter a solução-estoque com concentração de $5 \mathrm{~g} \mathrm{~L}^{-1}$. A solução-estoque foi preparada diariamente pouco antes de sua utilização e a solução extratora, na concentração desejada, somente no momento de seu uso. Esse procedimento teve a finalidade de evitar a hidrólise prévia do AITC.

Foram utilizados $5 \mathrm{~L}$ de cada solução por ponto de amostragem. À medida que as minhocas fugiam do interior do solo e atingiam a superfície, eram coletadas e lavadas rapidamente, e depois fixadas em etanol $70 \%$ por 4 horas (ISO/DIS 23611$1,2004)$. O procedimento de amostragem foi finalizado após 10 minutos da total infiltração da solução (TANCK et al., 2000; ZABORSKI, 2003). As minhocas extraídas em cada ponto foram acondicionadas em recipientes identificados de $250 \mathrm{~mL}$.

Quando ocorria amputação das minhocas durante a extração, ambas as partes eram coletadas para a avaliação da biomassa. Na determinação da densidade populacional, foi considerada apenas a parte frontal (ISO/DIS 23611-1, 2004).

Após a fixação, as minhocas foram transferidas para uma solução de formaldeído $40 \mathrm{~g}$ $\mathrm{L}^{-1}$ por nove dias e, em seguida, os animais foram armazenados em etanol $70 \%$ para preservação (Bouché e Gardner, 1984; ISO/DIS 23611-1, 2004). Essa solução foi substituída até a mesma permanecer límpida (ZABORSKI, 2003). 
A determinação da densidade populacional e da biomassa foi realizada com o material preservado no Laboratório de Biologia do Solo da UFPR. As minhocas foram lavadas em água deionizada com pisseta até completa remoção de resíduos aderidos à superfície do corpo. Em seguida, foram enxutas em papel toalha por, aproximadamente, um minuto, contadas e pesadas em uma balança com precisão de miligrama. Após, foram conservadas novamente em etanol $70 \%$. A identificação das minhocas coletadas foi realizada de acordo com SiMS e GERARD (1985), RighI (1990), SChwert (1990) e TALAVERa (1990).

O delineamento experimental foi inteiramente casualizado com doze tratamentos e sete repetições. Realizou-se análise fatorial de três doses de AITC (50, 100 e $150 \mathrm{mg} \mathrm{L}^{-1}$ ) e uma de formaldeído $2,2 \mathrm{~g} \mathrm{~L}^{-1}$ testadas em três diferentes sistemas. Os números de densidade populacional e de biomassa foram transformados para uma área de um metro quadrado sendo, em seguida, calculada a biomassa média. A biomassa média foi multiplicada por 100 para realização da análise de variância e do Teste de Tukey a 5\% de probabilidade.

\section{RESULTADOS E DISCUSSÃO}

\subsection{Densidade populacional, biomassa e biomassa média}

Pelo teste de Tukey a $5 \%$ de probabilidade (Tabela 3) observa-se que no sistema plantio direto, o tratamento com AITC 50 foi estatisticamente superior aos demais na obtenção da densidade populacional (144 ind $\mathrm{m}^{-2}$ ). Esse valor está próximo dos valores máximos verificados por Voss (1986), Peixoto e Marochi (1996) e Tanck et al. (2000) em municípios da região $\left(116,8,168\right.$ e 170,2 ind $\mathrm{m}^{-2}$ respectivamente).

$\mathrm{O}$ valor de biomassa estatisticamente superior foi alcançado com o tratamento AITC $100\left(31,3 \mathrm{~g} \mathrm{~m}^{-2}\right)$ (Tabela 3), que está dentro da faixa encontrada por Tanck et al. (2000) $\left(11,1\right.$ a 50,0 $\left.\mathrm{g} \mathrm{m}^{-2}\right)$. A espécie Amynthas gracilis é uma espécie epiendogêica que vive na camada de $0-15 \mathrm{~cm}$ e, dessa maneira, nesse talhão a solução de formaldeído pode ter provocado um aprofundamento ou afastamento do ponto de aplicação das minhocas quando de sua utilização, com posterior imobilização e morte (Righi, 1997; Chan e Munro, 2001; Zaborski, 2003). Porém, com O AITC 150 obteve-se a maior biomassa média.

Para o sistema pastagem perene, o tratamento com formaldeído 2,2 $\mathrm{g} \mathrm{L}^{-1}$ extraiu as maiores densidade populacional e biomassa total, diferindose estatisticamente dos demais. Os extratores AITC 100 e 150 foram estatisticamente equivalentes e alcançaram a maior biomassa média.
Ocorreu grande variação entre os resultados observados com o extrator formaldeído $2,2 \mathrm{~g} \mathrm{~L}^{-1}$ e as três doses de AITC testadas (50, 100 e $\left.150 \mathrm{mg} \mathrm{L}^{-1}\right)$. Esse fato deve ser devido, fundalmentalmente, à distribuição espacial das minhocas entre outras variáveis (EDWARDS, 1983; Edwards et al., 1995; Fragoso e LaVelle, 1992; Poier e Richter, 1992; CAllaham e Hendrix, 1997; JimÉnez et al., 2001). Por outro lado, o valor de densidade populacional verificado com AITC na dose de $50 \mathrm{mg}$ $\mathrm{L}^{-1}\left(81,6\right.$ ind $\left.\mathrm{m}^{-2}\right)$ está dentro da faixa obtida por BRowN et al. (2003), por coleta manual, em pastagem perene na região de Londrina (48-182 ind $\mathrm{m}^{-2}$ ).

Na mata, a maior densidade populacional foi obtida pela solução de formaldeído $2,2 \mathrm{~g} \mathrm{~L}^{-1}$ (38,7 ind m${ }^{2}$ ) e as doses de 50, 100 e $150 \mathrm{mg} \mathrm{L}^{-1}$ de AITC não diferiram estatisticamente entre si. Para a variável biomassa, os tratamentos formaldeído 2,2 $\mathrm{g} \mathrm{L}^{-1}$, AITC 50 e AITC 100 foram equivalentes estatisticamente e superiores ao AITC 150 (Tabela 3). CHAN e MunRo (2001) concluíram que o número de minhocas extraídas com a suspensão de mostarda aumentou com o incremento da concentração até determinado ponto, reduzindo a partir dele. Aqui esse comportamento foi observado na biomassa total e biomassa média, onde os maiores valores foram encontrados com AITC na dose de $100 \mathrm{mg} \mathrm{L}^{-1}$ (Tabela 3).

$$
\text { Ao se estabelecer a relação }
$$
clitelados:aclitelados extraídos com as diferentes soluções (Tabela 4), verifica-se que a solução de formaldeído extraiu maior número de indivíduos aclitelados no ecossistema plantio direto $(85,7 \%)$, seguido sucessivamente pela mata e pastagem perene. Por outro lado, a solução de AITC nas doses de 50, 100 e $150 \mathrm{mg} \mathrm{L}^{-1}$ extraiu o maior número de indivíduos aclitelados no ecossistema mata $(77,4$ a $93,7 \%)$. Esse ambiente é menos favorável ao crescimento e desenvolvimento das minhocas terrestres devido, fundamentalmente, à maior acidez (Tabela 1) e qualidade da M.O., e, por essa razão, a reprodução deve ser mais intensa para assegurar sua permanência (LAVELLE, 1983). Esse fato também pode ser observado na menor biomassa média obtida nesse ecossistema, com todos os extratores utilizados, em relação aos demais ecossistemas (Tabela 3).

Com o aumento na dose de AITC utilizada, houve redução no número de indivíduos aclitelados extraídos e incremento na biomassa média (Tabelas 3 e 4). O número de indivíduos jovens extraídos tende a reduzir com o incremento da concentração da solução extratora, pois são mais suscetíveis aos efeitos deletérios de produtos químicos que os adultos (Daniel et al. citados por Chan e MunRo, 2001). Dessa maneira, verifica-se que existem diferenças na obtenção de indivíduos em diferentes estádios de crescimento e desenvolvimento, em um mesmo período, em função do extrator utilizado (BOUCHÉ e GARDNER, 1984; LEE, 1985b; LAWRENCE e BOWERS, 2002; ZABORSKI, 2003). 
Tabela 3. Densidade populacional, biomassa e biomassa média de minhocas obtidas com os extratores formaldeído na dose de 2,2 $\mathrm{g} \mathrm{L}^{-1}$ e AITC nas doses de 50, 100 e $150 \mathrm{mg} \mathrm{L}^{-1}$ em três sistemas distintos (plantio direto, pastagem perene e mata), Castro (PR), 2003

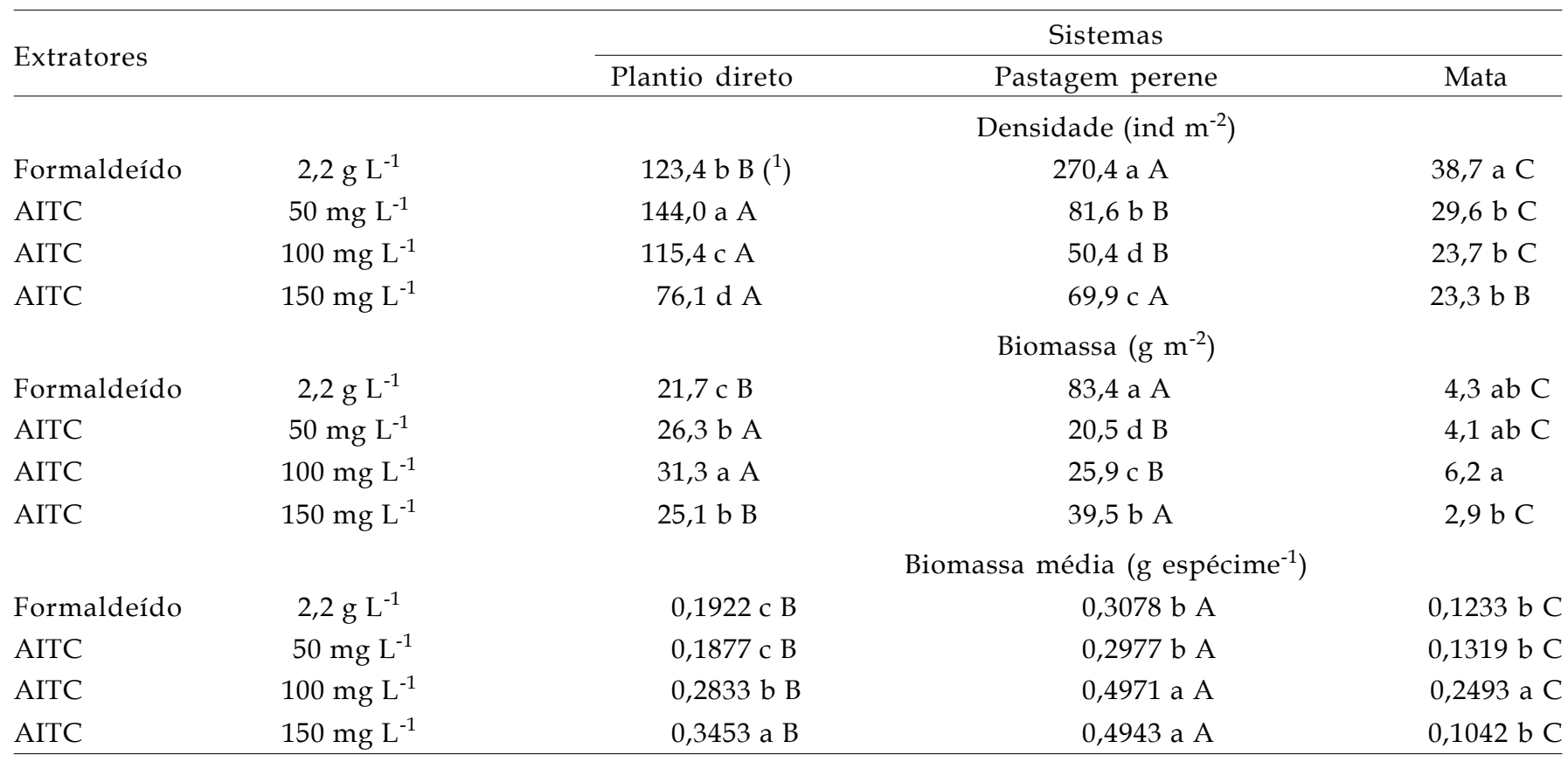

( $\left.{ }^{1}\right)$ Médias seguidas pela mesma letra minúscula, nas colunas(entre extratores) e maiúscula, nas linhas (entre sistemas) para a mesma variável, não diferem entre si pelo teste de Tukey $(p<0,05)$.

Tabela 4. Relação entre a densidade populacional dos indivíduos (minhocas) clitelados:aclitela-dos (em \%) obtida com os extratores formaldeído $2,2 \mathrm{~g} \mathrm{~L}^{-1}$ e AITC nas doses de 50, 100 e $150 \mathrm{mg} \mathrm{L}^{-1}$, Castro (PR), 2003

\begin{tabular}{lccc}
\hline Extratores & $\begin{array}{c}\text { Plantio } \\
\text { Direto }\end{array}$ & $\begin{array}{c}\text { Pastagem } \\
\text { perene }\end{array}$ & Mata \\
\hline Formaldeído 2,2 g L-1 & $14,3: 85,7$ & $27,8: 72,2$ & $21,6: 78,4$ \\
AITC $50 \mathrm{mg} \mathrm{L}^{-1}$ & $20,9: 79,1$ & $18,2: 81,8$ & $10,8: 89,2$ \\
AITC $100 \mathrm{mg} \mathrm{L}^{-1}$ & $32,2: 67,8$ & $36,6: 63,4$ & $22,6: 77,4$ \\
AITC $150 \mathrm{mg} \mathrm{L}^{-1}$ & $34,3: 65,7$ & $59: 41: 00$ & $6,3: 93,7$ \\
\hline
\end{tabular}

No sistema mata, observaram-se os menores valores de densidade populacional, biomassa e biomassa média entre todos os ecossistemas avaliados (Tabela 3). Esse ambiente estava totalmente modificado, com o menor número de espécies e nenhuma espécie nativa (Tabelas 5 e 6 ).

Apenas em sistemas cuja influência antropogênica não é significativa é que as espécies nativas têm oportunidade de permanecer, com as espécies exóticas invasoras sendo mais adaptadas a sítios antropogênicos (Fragoso et al., 1997, 1999b). TANCK et al. (2000) verificaram números muito

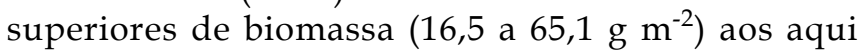

obtidos $\left(2,9\right.$ a 6,2 $\left.\mathrm{g} \mathrm{m}^{-2}\right)$ em uma mata nativa na região de Ponta Grossa (Tabela 3). As diferenças se devem, principalmente, ao fato de a mata nativa avaliada por TANCK et al. (2000) não ter influência antropogênica significativa nem estar sujeita a condições anaeróbias intermitentes, que eram características da área de mata objeto dessa pesquisa.

A menor biomassa média verificada no sistema mata foi com a utilização de AITC na dose de $150 \mathrm{mg} \mathrm{L}^{-1}\left(0,1042 \mathrm{~g}\right.$ espécime $\left.{ }^{-1}\right)$ (Tabela 3). Esse fato indica que aqui não existiam espécimens adultos de grande massa corpórea, fato confirmado pela menor relação clitelados:aclitelados $(6,3: 93,7)$ (Tabela 4).

Tabela 5. Ocorrência das diferentes famílias de minhocas nos ecossistemas avaliados, com os extratores formaldeído 2,2 $\mathrm{g} \mathrm{L}^{-1}$ e AITC nas doses 50, 10 e $150 \mathrm{mg}$ $\mathrm{L}^{-1}$, Castro (PR), 2003

\begin{tabular}{lcc}
\hline Famílias & Plantio Direto & Pastagem perene \\
\cline { 2 - 3 } & 1 & $\mathrm{~N}^{\mathrm{o}}$ de espécies \\
\cline { 2 - 3 } Lumbricidae & 0 & 1 \\
Octochaetidae & 4 & 1 \\
Megascolecidae & 4 & 4 \\
\hline
\end{tabular}


Tabela 6. Ocorrência das diferentes espécies de minhocas nos ecossistemas avaliados em função da solução extratora utilizada, Castro (PR), 2003

\begin{tabular}{lcccc}
\hline Espécies & Formaldeído & AITC 50 & AITC 100 & AITC 150 \\
\hline & $2,2 \mathrm{~g} \mathrm{~L}^{-1}$ & & $\mathrm{mg} \mathrm{L}^{-1}-$ & 0 \\
Allolobophora parva & $\mathrm{PD}, \mathrm{PP}$ & $\mathrm{PP}$ & $\mathrm{MT}, \mathrm{PP}$ & 0 \\
Dichogaster bolaui & $\mathrm{MT}$ & $\mathrm{PP}$ & $\mathrm{PD}$ & $\mathrm{PD}, \mathrm{PP}$ \\
Metaphire californica & $\mathrm{PP}$ & $\mathrm{PD}, \mathrm{PP}, \mathrm{MT}$ & $\mathrm{PD}, \mathrm{PP}$ & $\mathrm{PD}, \mathrm{PP}$ \\
Amynthas morrisi & $\mathrm{PD}, \mathrm{PP}, \mathrm{MT}$ & $\mathrm{PD}, \mathrm{PP}$ & $\mathrm{PD}, \mathrm{PP}$ & $\mathrm{PD}, \mathrm{PP}$ \\
Amynthas gracilis & $\mathrm{PP}$ & $\mathrm{PP}, \mathrm{MT}$ & $\mathrm{MT}$ & $\mathrm{PD}, \mathrm{PP}, \mathrm{MT}$ \\
Amynthas corticis & $\mathrm{PP}, \mathrm{MT}$ & & &
\end{tabular}

PD(plantio direto); PP (pastagem); MT (mata)

* Não extraída com o respectivo extrator.

$\mathrm{Na}$ tabela 3, verifica-se que a biomassa média das minhocas extraídas com a solução de AITC na dose de $100 \mathrm{mg} \mathrm{L}^{-1}$, em todos os ecossistemas avaliados, foi estatisticamente superior à solução de formaldeído 2,2 $\mathrm{g} \mathrm{L}^{-1}$. A solução de AITC na dose de $100 \mathrm{mg} \mathrm{L}^{-1}$ também extraiu maior número de indivíduos clitelados em relação à solução de formaldeído $2,2 \mathrm{~g} \mathrm{~L}^{-1}$ (Tabela 4). Em Zaborski (2003), a extração com a solução de AITC na dose de $100 \mathrm{mg} \mathrm{L}^{-1}$ foi mais eficiente na amostragem de minhocas anécicas de maior biomassa média (Lumbricus terrestris). Dessa forma, as minhocas de maior biomassa, ao contrário do que ocorre com a solução de formaldeído, são direcionadas à superfície quando da utilização de AITC, enquanto a solução de formaldeído leva a um aprofundamento no solo ou afastamento dessas minhocas do ponto de aplicação e, posteriormente, é provável que provoque sua inativação e morte.

\subsection{Famílias de minhocas terrestres}

As minhocas adultas da família Megascolecidae (Amynthas morrisi, A. gracilis, A. corticis e Metaphire californica) predominaram nos ecossistemas estudados (Tabelas 5 e 6). No talhão de plantio direto, corresponderam a $98,6 \%$, sendo o restante espécimens adultos da família Lumbricidae (Allolobophora parva). Os indivíduos adultos de Amynthas morrisi representavam 93,5\% das minhocas extraídas nesse talhão.

$\mathrm{Na}$ região de Castro, onde a temperatura média anual $\left(17\right.$ a $\left.18^{\circ} \mathrm{C}\right)$ e a não ocorrência de estação seca no inverno (junho a agosto com 250 a $350 \mathrm{~mm}$ de precipitação) tendem a manter a cobertura do solo em plantio direto, o ambiente edáfico é mantido favorável a Amynthas spp. e Metaphire spp. (Fragoso et al., 1999a; Brown et al., 2003). Em oposição, na região de Londrina as minhocas presentes em florestas perturbadas e em sistemas diversos são, principalmente, das famílias Octochaetidae e Glossoscolecidae (Brown et al., 2003). O clima mais quente nessa região, onde a temperatura média anual fica em torno de $21^{\circ} \mathrm{C}$ e a precipitação pluvial na estação seca é ao redor de $225 \mathrm{~mm}$ (de junho a agosto), tende a reduzir o teor de M.O. na superfície do solo, limitando a colonização e sobrevivência de Amynthas spp. e Metaphire spp. (IAPAR, 2000; Fragoso et al., 1999b; BROWn et al., 2003). Nos espécimens dessas famílias encontrados na região de Londrina, a biomassa média é inferior às dos Megascolecidae Amynthas spp. e Metaphire spp. e, por essa razão, a densidade populacional de minhocas nessa região é similar à da região de Castro, mas a biomassa é muito menor.

Não foi encontrada nenhuma espécie nativa, principalmente da família Glossoscolecidae (RIGHI, 1997), em nenhum dos ecossistemas avaliados (Tabela 5). Todas as espécies de Megascolecidae e Lumbricidae são exóticas. Dessa maneira, é de se presumir que esses ambientes estão completamente transformados, não possuindo mais nenhuma característica original. A espécie Dichogaster bolaui da família Octochaetidae, observada nos ecossistemas pastagem perene e mata, tem origem no continente africano (RIGHI, 1990; FRAGOSO et al., 1999a; BLAKEMORE, 2003).

Nos sistemas amostrados (plantio direto e pastagem perene), foram encontradas cinco espécies diferentes de minhocas no talhão de plantio direto e seis espécies na pastagem perene (Tabela 5), com predomínio de epiendogêicas, indicando haver em ambos os sistemas potencial similar para abrigar diferentes espécies. Normalmente, mesmo nas comunidades mais diversas em uma área agrícola, são encontradas de 4 a 6 espécies diferentes de minhocas e raramente mais de 10 (Lee citado por Edwards et al., 1995). Cada espécie de minhoca tem influência específica nos processos do solo (ciclagem de nutrientes, estabilidade dos agregados e macroporosidade). 
$\mathrm{Na}$ tabela 6, observa-se que as diferentes espécies dos ecossistemas estudados se comportam de forma diferente em função da solução extratora utilizada (RAW, 1959; BOUChÉ e GARDNER, 1984; Lee, 1985b; LAWRENCE e BOWERS, 2002; ZABORSKI, 2003). Deve ser ressaltado que com a solução de formaldeído, o extrator mais empregado na avaliação da densidade populacional e biomassa de minhocas, foram extraídas apenas duas espécies de minhocas terrestres (Allolobophora parva e Amynthas morrisi) de um total de cinco espécies encontradas no talhão de plantio direto com os extratores testados (Tabelas 5 e 6 ).

A espécie Amynthas gracilis só foi extraída no talhão de plantio direto com as três doses de AITC testadas; Amynthas corticis com AITC na dose de 150 $\mathrm{mg} \mathrm{L}^{-1}$; Metaphire californica foi extraída nesse mesmo talhão apenas com a solução de AITC nas doses de 100 e $150 \mathrm{mg} \mathrm{L}^{-1}$ (Tabela 6).

No talhão de pastagem perene, $95 \%$ das minhocas adultas presentes eram da família Megascolecidae e estavam distribuídas nas seguintes proporções: Amynthas morrisi 45,9\%, A. gracilis 39,3 $\%$, A. corticis $5,7 \%$ e Metaphire californica $4,1 \%$ dos indivíduos adultos. Uma espécie da família Lumbricidae (Allolobophora parva) estava presente com 4,1 \% dos indivíduos adultos e 0,9 \% eram indivíduos da espécie Dichogaster bolaui.

Apenas com o extrator AITC na dose de 100 mg L ${ }^{-1}$ foi extraída a espécie Dichogaster bolaui no talhão de pastagem perene. Esse mesmo extrator foi o único que não extraiu a espécie Metaphire californica nesse mesmo talhão (Tabela 6). Por essa razão, a utilização de diferentes extratores em um mesmo talhão pode ampliar os resultados alcançados. Da mesma forma, não se pode afirmar com certeza que determinada espécie de minhoca não ocorre em um talhão em função das variações encontradas na reação de diferentes espécies a diferentes soluções extratoras (RAW, 1959; Bouché e Gardner, 1984; Lee, 1985b; Chan e Munro, 2001; LAWRENCE e BOWERS, 2002; ZABORSKI, 2003). Também deve ser considerada a distribuição espacial das manchas de espécies diferentes de minhocas, que podem ocupar nichos distintos ou parcialmente comuns (EDWARdS et al., 1995; JimÉneZ et al., 2001).

\section{CONCLUSÕES}

1. No sistema plantio direto, o extrator AITC na dose de $50 \mathrm{mg} \mathrm{L}^{-1}$ é de maior eficácia na obtenção da densidade populacional e biomassa que o extrator formaldeído $2,2 \mathrm{~g} \mathrm{~L}^{-1}$.

2. O extrator formaldeído $2,2 \mathrm{~g} \mathrm{~L}^{-1}$ é mais eficiente nos sistemas pastagem perene e mata.
3. O aumento na dose de AITC resulta em redução na eficiência do extrator.

4. A região estudada é caracterizada pelo predomínio de espécies exóticas de minhocas, principalmente da família Megascolecidae.

\section{AGRADECIMENTOS}

Ao Sr. Roelof Rabbers, proprietário da Fazenda Onça (Castro, PR), pela oportunidade de realização dessa pesquisa em sua propriedade e ao Rolinho, pelo auxílio remunerado na extração das minhocas.

\section{REFERÊNCIAS}

BLAKEMORE, R.J. Japanese earthworms (Annelida: Oligochaeta): a review and checklist of species. Organisms Diversity \& Evolution, v.03, p.241-244, 2003.

BOREK, V.; MORRA, M.J.; BROWN, P.D.; McCAFFREY, J.P. Transformation of the glucosinolate-derived allelochemicals allyl isothiocyanate and allylnitrile in soil. Journal of Agricultural and Food Chemistry,Washington, v.43, p.19351940, 1995.

BOUCHÉ, M.B.; GARDNER, R.H. Earthworm functions: VIII. - population estimation techniques. Revue d'Ecologie et Biologie du Sol, Montrouge, v.21, p.37-63, 1984.

BROWN, G.G.; BENITO, N.P.; PASINI, A.; SAUTTER, K.D.; GUIMARÃES, M.F.; TORRES, E. No-tillage greatly increases earthworm populations in Paraná state, Brazil. Pedobiologia, Jena, v.47, p.764-771, 2003.

CALLAHAM, M.A.; HENDRIX, P.F. Relative abundance and seasonal activity of earthworms (Lumbricidae and Megascolecidae) as determined by hand-sorting and formalin extraction in forest soils on southern Appalachian Piedmont. Soil Biology and Biochemistry, Oxford, v.29, p.317-321, 1997.

CHAN, K.Y.; MUNRO, K. Evaluating mustard extracts for earthworm sampling. Pedobiologia, Jena, v.45, 272-278, 2001.

EDWARDS, C.A. Earthworm ecology in cultivated soils. In: SATCHELL, J.E., (Ed.). Earthworm Ecology from Darwin to Vermiculture. London: Chapman and Hall, 1983. p.123-138.

EDWARDS, C.A.; BOHLEN, P.J.; LINDEN, D.R.; SUBLER, S. Earthworms in agroecosystems. In: HENDRIX, P.F. (Ed.). Earthworm Ecology and Biogeography in North America. Boca Raton: CRC, 1995. p.185-213.

FASOLO, P.J.; CARVALHO, A.P.; POTTER, R.O.; BOGNOLA, I.A.; BHERING, S.B.; MARTORANO, L.G. Caracterização dos Solos do Município de Castro, PR. Rio de Janeiro: EMBRAPA - CNPS, 2002. 88p. (Boletim de Pesquisa e Desenvolvimento n.09). 
FRAGOSO, C.; LAVELLE, P. Earthworm communities of tropical rain forests. Soil Biology and Biochemistry, Oxford, v.24, p.1397-1408, 1992.

FRAGOSO, C.; BROWN, G.G.; PATRÓN, J.C. BLANCHART, E.; LAVELLE, P.; PASHANASI, B.; SENAPATI, B.; KUMAR, T. Agricultural intensification, soil biodiversity and agroecosystem function in the tropics: the role of earthworms. Applied Soil Ecology, v.06, p.17-35, 1997.

FRAGOSO, C.; KANYONYO, J.; MORENO, A.;SENAPATI, B.K.; BLANCHART, E.; RODRIGUEZ, C. A survey of tropical earthworms: taxonomy, biogeography and environmental plasticity. In: LAVELLE, P.; BRUSSAARD, L.; HENDRIX, P., (Ed.). Earthworm Management in Tropical Agroecosystems. Oxon: CAB International, 1999a. p.01-25.

FRAGOSO, C.; LAVELLE, P.; BLANCHART, E.; SENAPATI, B.K.; JIMENEZ, J.J.; MARTINEZ, M.A.; DECAËNS, T.; TONDOH, J. Earthworm communities of tropical agroecosystems: origin, structure and influence of management practices. In: LAVELLE, P.; BRUSSAARD, L.; HENDRIX, P. (Ed.). Earthworm Management in Tropical Agroecosystems. Oxon: CAB International, 1999b. p.27-55.

GUNN, A. The use of mustard to estimate earthworm populations. Pedobiologia,Jena, v.36, p.65-67, 1992.

INSTITUTO AGRONÔMICO DO PARANÁ - IAPAR. Cartas Climáticas do Paraná. Versão 1.0. Londrina: IAPAR, 2000. CDROM.

INTERNATIONAL

ORGANIZATION

FOR

STANDARDIZATION - ISO/DIS 23611-1. Soil Quality: sampling of soil invertebrates. Part. Hand-sorting and formalin extraction of earthworms. Geneva: International Organization for Standardization, 2004. 18p.

JIMÉNEZ, J.J.; ROSSI, J.P.; LAVELLE, P. Spatial distribution of earthworms in acid-soil savannas of the eastern plains of Colombia. Applied Soil Ecology, v.17, p.267-278, 2001.

LAVELLE, P. The structure of earthworm communities. In: SATCHELL, J.E., (Ed.). Earthworm Ecology: from Darwin to vermiculture. London: Chapman and Hall, 1983. p.449-466.

LAWRENCE, A.P.; BOWERS, M.A. A test of the "hot" mustard extraction method of sampling earthworms. Soil Biology and Biochemistry, Oxford, v.34, p.549-552, 2002.

LEE, K.E. Populations and associations. In: LEE, K.E., (Ed.). Earthworms: their ecology and relationships with soils and land use. Florida: Academic Press, 1985a. p.89-134.

LEE, K.E. Appendix: field sampling methods. In: LEE, K.E., (Ed.). Earthworms: their ecology and relationships with soils and land use. Florida: Academic Press, 1985b. p.331-349.

MACHADO, M.A.M. Procedimentos metodológicos em física dos solos relacionados ao manejo dos solos. In: LIMA, M.R.; SIRTOLI, A.E.; SERRAT, B.M.; WISNIEWSKI, C.; ALMEIDA, L.; MACHADO, M.A.M.; MARQUES, R.; MOTTA, A.C.V.; KRIEGER, K.I.; OLIVEIRA, A.C.; FERREIRA, F.V. (Ed.). Manual de Diagnóstico da Fertilidade e Manejo dos Solos Agrícolas. 2.ed. Curitiba: UFPR, 2003. p.103-122.
MARQUES, R.; MOTTA, A.C.V. Análise química de solo para fins de fertilidade. In: LIMA, M.R.; SIRTOLI, A.E.; SERRAT, B.M.; WISNIEWSKI, C.; ALMEIDA, L.; MACHADO, M.A.M.; MARQUES, R.; MOTTA, A.C.V.; KRIEGER, K.I.; OLIVEIRA, A.C.; FERREIRA, F.V. (Ed.). Manual de Diagnóstico da Fertilidade e Manejo dos Solos Agrícolas. 2.ed. Curitiba: UFPR, 2003. p.81-102.

PAOLETTI, M.G. The role of earthworms for assessment of sustainability and as bioindicators. Agriculture, Ecossystems and Environment, Amsterdam, v.74, p.137-155, 1999.

PEIXOTO, R.T.G.; MAROCHI, A.I. A influência da minhoca Pheretima sp. nas propriedades de um Latossolo Vermelho escuro álico e no desenvolvimento de culturas em sistema de plantio direto em Arapoti - PR. Revista Plantio Direto, Passo Fundo, v.35, p.23-25, 1996.

POIER, K.R.; RICHTER, J. Spatial distribution of earthworms and soil properties in an arable loess soil. Soil Biology and Biochemistry, Oxford, v.24, p.1601-1608, 1992.

RAW, F. Estimating earthworm populations by using formalin. Nature, v.184, p.1661-1662, 1959.

RIGHI, G.R. Minhocas de Mato Grosso e de Rondônia. Brasília: CNPq, 1990. 157p.

RIGHI, G.R. Minhocas da América Latina: diversidade, função e valor. In: CONGRESSO BRASILEIRO DE CIÊNCIA DO SOLO, 26., Rio de Janeiro, 1997. Resumos Expandidos. Rio de Janeiro, Sociedade Brasileira de Ciência do Solo, 1997. CD-ROM.

RUF, A.; BECK, L.; DREHER, P.; HUND-RINKE, K.; RÖMBKE, J.; SPELDA, J. A biological classification concept for the assessment of soil quality: "biological soil classification scheme" (BBSK). Agriculture, Ecossystems and Environment, Amsterdam, v.98, p.263-271, 2003.

SCHMIDT, O.; CURRY, J.P.; HACKETT, R.A.; PURVIS, G.; CLEMENTS, R.O. Earthworm communities in conventional wheat monocropping and low-input wheat-clover intercropping systems. Annals of Applied Biology, Wellesbourne, v.138, p.377-388, 2001.

SCHWERT, D.P. Oligochaeta: Lumbricidae. In: DINDAL, D.L., (Ed.). Soil Biology Guide. New York: John Wiley \& Sons, 1990. p.341-356.

SIMS, R.W.; GERARD, B.M. Family Megascolecidae. In: SIMS, R.W.; GERARD, B.M. (Ed.). Earthworms - Synopsis of the British Fauna. London: The Linnean Society of London, 1985. p.126-136.

SINNAEVE, J. Foreword. In: LAVELLE, P.; BRUSSAARD, L.; HENDRIX, P., (Ed.). Earthworm Management in Tropical Agroecosystems. Oxon: CAB International, 1999. p.x-xi.

TALAVERA, J.A. Claves de identificación de las lombrices de tierra (Annelida: Oligochaeta) de Canarias. Vieraea, v.18, p.113119, 1990. 
TANCK, B.; SANTOS, H.R.; DIONÍSIO, J.A. Influência de diferentes sistemas de uso e manejo do solo sobre a flutuação populacional do Oligochaeta edáfico Amynthas spp.. Revista Brasileira de Ciência do Solo, Viçosa, v.24, p.409-415, 2000.

VOSS, M. População de minhocas em diferentes sistemas de plantio. Revista Plantio Direto, Passo Fundo, v.17, p.06-07, 1986.
ZABORSKI, E.R. Allyl isothiocyanate: an alternative chemical expellant for sampling earthworms. Applied Soil Ecology, v.22, p.87-95, 2003. 